\title{
Thermometry of Fermionic Atoms in an Optical Lattice
}

\author{
Michael Köhl \\ Institute of Quantum Electronics, ETH Zürich Hönggerberg, CH-8093 Zürich, Switzerland
}

\begin{abstract}
Low temperatures are necessary for the observation of strongly correlated quantum phases of fermionic atoms in optical lattices. We analyze how the temperature of a Fermi gas is altered when the fermions are loaded into an optical lattice with an underlying harmonic confining potential and show how the temperature can be measured. The temperature of the atoms in the optical lattice determines the fraction of doubly occupied lattice sites of a two-component Fermi gas. We analytically calculate this quantity and find a strong temperature dependence. This fraction can be measured by studying the production of molecules in the lattice using a Feshbach resonance which allows for precise thermometry of atoms in an optical lattice.
\end{abstract}

PACS numbers: 03.75.Ss, 05.30.Fk, 71.10.Ca

Ultracold fermionic atoms in optical lattices are a promising tool to simulate problems from condensed matter physics. Interesting phase transitions between many-body quantum phases in the lattice are predicted [1, 2]. For these phase transitions the temperature is one of the key control parameters and a precise knowledge of the temperature of the atoms in the lattice is necessary to experimentally determine the phase diagram. Proposals for cooling the atoms in the lattice in order to reach interesting quantum phases such as the BEC-BCS transition in lattices or an anti-ferromagnetic phase have been devised [1, 3, 4]. However, standard methods for thermometry - such as observing the rounding-off of the Fermi edge [5] - fail in the inhomogeneous lattice since they turned out to be dominated by the trapping potential rather than by temperature [6, 7].

Previous theoretical investigations of ultracold fermions in optical lattices have often neglected the role of the harmonic confining potential. For ongoing experiments, this confining potential is an unavoidable ingredient and its consequences, such as an inhomogeneous filling of the lattice and the coexistence of insulating and conducting regions, must be considered. In a typical experimental sequence a quantum degenerate Fermi gas is initially prepared in a purely harmonic optical dipole potential and subsequently transferred into a threedimensional optical lattice with underlying confining potential [7].

The transfer of a Fermi gas from a harmonic trapping potential into an optical lattice leads to a significant modification of the density of states. A change in the density of states can result in both an adiabatic cooling or an adiabatic heating of the gas. We find that for the situation realized in the experiments the gas is adiabatically heated which poses a difficulty for future experiments aiming at very low temperatures. To determine the temperature in the lattice we analyze the fraction of doubly occupied sites of a noninteracting two-component Fermi gas [8]. This can be measured experimentally by studying the formation of dimers in the optical lattice on a Feshbach resonance [9] or using photo association [10, 11, 12]. We calculate the fraction of doubly occupied sites analytically and find a strong temperature dependence which makes it ideal for thermometry of ultracold fermions in an optical lattice.

A noninteracting two-component Fermi gas in an optical lattice with an underlying harmonic confining potential can be described by the Hamiltonian

$$
H_{\sigma}=-t \sum_{<j, l>} c_{j \sigma}^{\dagger} c_{l \sigma}+\frac{m}{2} \omega^{2} d^{2} \sum_{j} j^{2} n_{j \sigma} .
$$

Here $\sigma=\{\uparrow, \downarrow\}$ denotes the spin of the particle, $t$ denotes the hopping matrix element between adjacent lattice sites, $c_{j \sigma}^{\dagger}$ and $c_{j \sigma}$ are the creation and the annihilation operators on the lattice site $j$, respectively, and $n_{j \sigma}=c_{j \sigma}^{\dagger} c_{j \sigma}$ is the number operator. Furthermore, $m$ denotes the atomic mass, $\omega$ the trapping frequency of the harmonic confining potential and $d=\lambda / 2$ the lattice spacing given by the laser wavelength $\lambda$. The interactions between atoms in different spin states are assumed to be zero, as realized experimentally by using a Feshbach resonance [7]. The trapping frequency $\omega$ of the confining potential is determined by the potential depth $V$ and the waist $w$ of the Gaussian intensity profile of the laser beam. For a three-dimensional lattice generated by three mutually orthogonal laser beams of identical waist $w$ and potential depth $V$ one obtains $\frac{m}{2} \omega^{2}=\frac{4 V}{w^{2}}$.

Let us first consider the single particle density of states [13] in the low-tunnelling limit in a three-dimensional optical lattice. There the particles are localized in the potential wells and we restrict ourselves to a single band approximation which is justified by the experiment [7]. For determining the density of states we assume the width of the lowest Bloch band $4 t$ to be smaller than the energy offset between neighboring lattice sites due to the harmonic confinement. This approximation is valid except for a very small region at the center of the harmonically trapped cloud, where delocalized atoms should be considered. However, only few atoms are affected and we neglect their contribution in the analytical calculation. The presence of the harmonic confinement modifies the density of states significantly: for a particle on the lattice site $j$, the potential energy is $\frac{m}{2} \omega^{2}\left(\frac{\lambda}{2}\right)^{2} j^{2}$. Allowing for only one particle per lattice site, the density of states in three dimensions reads

$$
\rho_{3 D}(E)=2 \pi E^{1 / 2}\left(\frac{m \omega^{2} \lambda^{2}}{8}\right)^{-3 / 2} .
$$

This shows that the density of states of fermions localized in an optical lattice with underlying harmonic confinement has the same power law dependence as that of free fermions. This analogy can be understood by the following argument: 
in free space the fermions are characterized by their momentum $k$ and the eigenstates are equally spaced in momentum space. Each fermion has a kinetic energy $E \propto k^{2}$. In the low-tunnelling limit with harmonic confinement the fermions occupy equally spaced lattice sites at positions $j \lambda / 2$ and have a potential energy $E \propto j^{2}(\lambda / 2)^{2}$ only, which results in the same dependence of the density of states on $E$ as in the free fermion case. From the density of states we directly obtain the Fermi energy in the tight-binding limit:

$$
E_{F}(N)=\frac{m \omega^{2} \lambda^{2}}{8}\left(\frac{3 N}{4 \pi}\right)^{2 / 3} .
$$

In one and two dimensions we find the same analogy to the free fermion case, namely $\rho_{2 D}(E)=8 \pi /\left(m \omega^{2} \lambda^{2}\right)$ and $\rho_{1 D}(E)=\left(m \omega^{2} \lambda^{2} E / 8\right)^{-1 / 2}$, or generally $\rho(E) \propto E^{d / 2-1}$ with $d$ being the dimension.

To estimate the validity of the low-tunnelling approximation we compare the analytical results with a numerical calculation where we diagonalize the Hamiltonian (1) exactly. We compute the density of states and fit the result with a function $\widetilde{\rho}(E)=a \cdot E^{\nu}$ to obtain the exponent $\nu$. For a large potential depth, the value of $\nu$ approaches $1 / 2$, as expected from equation (2). Similarly, for a two-dimensional quantum gas in an optical lattice the exponent approaches 0 . The calculations were performed on $300^{2}$ lattice sites in $2 \mathrm{D}$ and $100^{3}$ lattice sites in 3D.

When atoms are loaded into an optical lattice the density of states changes from the three-dimensional harmonic oscillator result $\rho(E) \propto E^{2}$ to the low-tunneling results $\rho(E) \propto$ $E^{d / 2-1}$. Adiabatically changing the density of states of a system can be used to cool or heat a gas, provided that collisions keep the gas in thermal equilibrium [14, 15]. We assume an adiabatic evolution of the gas during the loading procedure which implies that no heat is exchanged with the environment. The interactions between the atoms are supposed to be sufficiently large to ensure thermalization of the sample but small enough so that the energy spectrum of the gas is not altered. In such a situation the gas evolves along a path of constant entropy $S$. The entropy $S$ of a Fermi gas is given by [16]

$$
\frac{S}{k_{B}}=\frac{\mathcal{E}-\mu N}{k_{B} T}+\sum_{n} \log \left(1+e^{\left(\mu-E_{n}\right) / k_{B} T}\right)
$$

with the total energy $\mathcal{E}=\int_{-\infty}^{\infty} E \rho(E) f(E) d E$, the chemical potential $\mu$ and the temperature $T$. $n$ labels the energy eigenstates $E_{n}$ and $f(E)=\left(e^{(E-\mu) / k_{B} T}+1\right)^{-1}$ is the Fermi distribution function. The chemical potential is determined from the normalization to the total particle number $N=\int_{-\infty}^{\infty} \rho(E) f(E) d E$. For low temperatures the entropy in the two limiting cases can be obtained using the Sommerfeld approximation. For noninteracting fermions trapped in a three-dimensional power-law potential $V(\mathbf{r}) \propto \sum_{m=x, y, z} r_{m}^{\alpha}$ the entropy is given by [17, 18

$$
\frac{S}{k_{B}}=N \pi^{2}\left(\frac{1}{\alpha}+\frac{1}{2}\right) \frac{k_{B} T}{E_{F}}+\mathcal{O}\left(\left(\frac{k_{B} T}{E_{F}}\right)^{2}\right) .
$$

We now compare the result of the entropy for a given number of particles in a harmonic trap $(\alpha=2)$ with an ideal Fermi gas in an optical lattice with underlying harmonic confinement. As discussed above, the latter has the same density of states as free fermions in a box potential and therefore corresponds to $\alpha=\infty$. Consequently, the entropy in a harmonic trap is a factor of 2 larger than for the fermions in the optical lattice. Thus when the transfer from the harmonic potential into the lattice is performed adiabatically it is accompanied by a temperature increase by a factor 2 . The effect of the turn-on of the lattice on the atom number statistics at the lattice sites has been calculated for a one-dimensional Fermi gas [19].

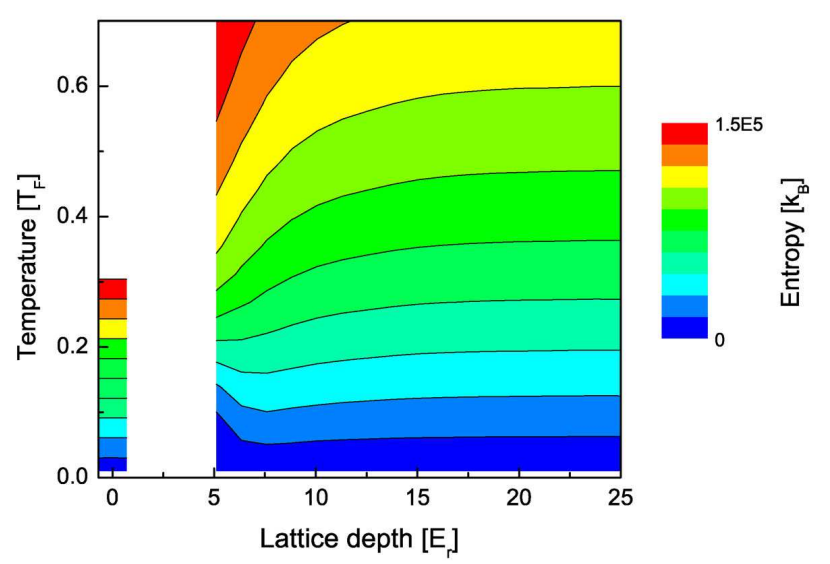

FIG. 1: Curves of constant entropy in the lattice. The calculation is performed by diagonalizing Hamiltonian 11 with $N=50000$ atoms trapped in three-dimensional optical lattice with the waist of the lattice laser being $w=70 \mu \mathrm{m}$, according to the situation in [9]. The values shown at zero lattice depth are the harmonic oscillator result without lattice. For $s=V / E_{r} \lesssim 5$ the tight-binding Hamiltonian may give incorrect results and therefore we refrain from numerical calculations in this regime.

We numerically calculate $S$ for various temperatures and lattice depths (see fig. 1), similar to what has been done previously for the homogeneous lattice [3]. For a large potential depth the numerical calculations agree with the analytical prediction of equation (5). Moreover, when moving along a line of constant entropy as the lattice depth is increased we observe that the temperature never drops below its initial value in the harmonic oscillator trap. This implies that no cooling can be achieved during the transfer into the lattice.

The quest for obtaining ultra-low temperatures in the optical lattice is accompanied with the need for precise thermometry. We show that the temperature can be obtained from the fraction of doubly occupied sites of a noninteracting twocomponent Fermi gas. A similar problem has been studied numerically for bosonic [20] and fermionic [8] atoms. We analytically calculate the number of doubly occupied sites $N_{2}$ assuming that both species are distributed according to the same Fermi distribution function $f(E)=f_{\uparrow}(E)=f_{\downarrow}(E)$

$$
N_{2}=\int_{-\infty}^{\infty} \rho(E) f^{2}(E) d E
$$


In two dimensions (i.e. for $\nu=0$ ) simple analytic expressions are obtained. We consider a Fermi gas strongly confined along one axis to create a two-dimensional quantum gas which is subject to two crossed optical lattices in the transverse directions. The chemical potential as a function of temperature is $\mu=k_{B} T \ln \left(e^{E_{F} / k_{B} T}-1\right)$ and the fraction of doubly occupied sites is

$$
n_{2}=\frac{N_{2}}{N}=\frac{k_{B} T}{E_{F}}\left(e^{-E_{F} / k_{B} T}+\frac{E_{F}}{k_{B} T}-1\right) .
$$

a)

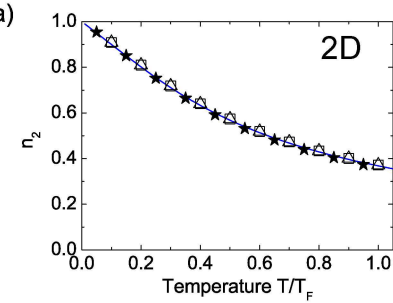

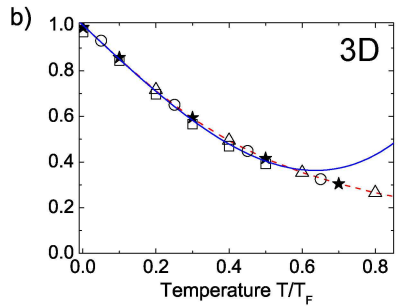

FIG. 2: a) Comparison of the analytical formula (7) (solid line) with the numerically calculated double occupation of a two-dimensional quantum gas in an optical lattice for various parameters by diagonalizing Hamiltonian (1). Squares: $s=25, w=70 \mu \mathrm{m}, N=5000$, stars: $s=15, w=100 \mu \mathrm{m}, N=3500$, triangles: $s=15, w=50 \mu \mathrm{m}, N=2500$. b) Comparison of the analytical formula 12 (solid line) with the numerically calculated occupation in a $3 \mathrm{D}$ optical lattice for various experimentally relevant parameters by diagonalizing Hamiltonian (1). Circles: $s=15, w=50 \mu \mathrm{m}, N=25000$, triangles: $s=20$, $w=70 \mu \mathrm{m}, N=15000$, squares: $s=15, w=100 \mu \mathrm{m}, N=40000$, stars: $s=25, w=130 \mu \mathrm{m}, N=25000$, triangles: $s=20, w=70 \mu \mathrm{m}, N=15000$. The dashed line is a numerical solution of equation 6 for the 3D lattice using the density of states given by eq. (2).

For the general case, we use a low-temperature expansion similar to the Sommerfeld expansion for free electrons. We integrate eq. (6) by parts and expand $R(E)=\int \rho(E) d E$ in a power series around $E=\mu$, analogous to the method described in [21]. We obtain the number of doubly occupied lattice sites to be

$$
N_{2}=\int_{-\infty}^{\mu} \rho(E) d E+\left.\sum_{n=1}^{\infty} a_{n}\left(k_{B} T\right)^{n} \frac{d^{n-1} \rho(\epsilon)}{d \epsilon^{n-1}}\right|_{\epsilon=\mu}
$$

with the coefficients $a_{n}$ defined by

$$
a_{n}=\int_{-\infty}^{\infty} \frac{x^{n}}{n !}\left(-\frac{d}{d x} \frac{1}{\left(e^{x}+1\right)^{2}}\right) d x
$$

and given by $a_{n}=\left(2-2^{2-n}\right) \zeta(n)$ for even values of $n$ and $a_{n}=-a_{n-1}$ for odd values of $n$. The Sommerfeld expansion yields $N=\int_{-\infty}^{\mu} \rho(E) d E+\left.\sum_{n=1}^{\infty} b_{2 n}\left(k_{B} T\right)^{2 n} \frac{d^{2 n-1} \rho(\epsilon)}{d \epsilon^{2 n-1}}\right|_{\epsilon=\mu}$ [21] with the coefficients $b_{2 n}=\int_{-\infty}^{\infty} \frac{x^{2 n}}{(2 n) !}\left(-\frac{d}{d x} \frac{1}{e^{x}+1}\right) d x=$ $a_{2 n}$, which represent the even terms in the expansion (8). The analytic expression for the fraction of doubly occupied lattice sites is then given by

$$
n_{2}=\frac{N_{2}}{N}=1+\left.\frac{1}{N} \sum_{n=0}^{\infty} a_{2 n+1}\left(k_{B} T\right)^{2 n+1} \frac{d^{2 n} \rho(\epsilon)}{d \epsilon^{2 n}}\right|_{\epsilon=\mu} .
$$

For a density of states $\rho \propto E^{\nu}$ the fraction of doubly occupied sites is given to first order in $k_{B} T / E_{F}$ by

$$
n_{2}=1-(\nu+1) \frac{k_{B} T}{E_{F}}+\mathcal{O}\left(\left(\frac{k_{B} T}{E_{F}}\right)^{2}\right) .
$$

This expression shows that there is a linear dependence of the fraction of doubly occupied sites on temperature in the degenerate regime $k_{B} T \ll E_{F}$. The slope depends only on the exponent of the density of states, but not on lattice depth (see figure 2). This makes this quantity ideally suited for thermometry.

For the special case of a deep three-dimensional optical lattice with $\nu=1 / 2$ (see equation (2) ) we use the known expression for the chemical potential $\mu=E_{F}\left(1-\frac{\pi^{2}}{12}\left(\frac{k_{B} T}{E_{F}}\right)^{2}\right)$ and expand $n_{2}$ to third order in $k_{B} T / E_{F}$ :

$$
n_{2}=1-\frac{3}{2} \frac{k_{B} T}{E_{F}}+\frac{\pi^{2}}{8}\left(\frac{k_{B} T}{E_{F}}\right)^{3}+\mathcal{O}\left(\left(\frac{k_{B} T}{E_{F}}\right)^{4}\right) .
$$

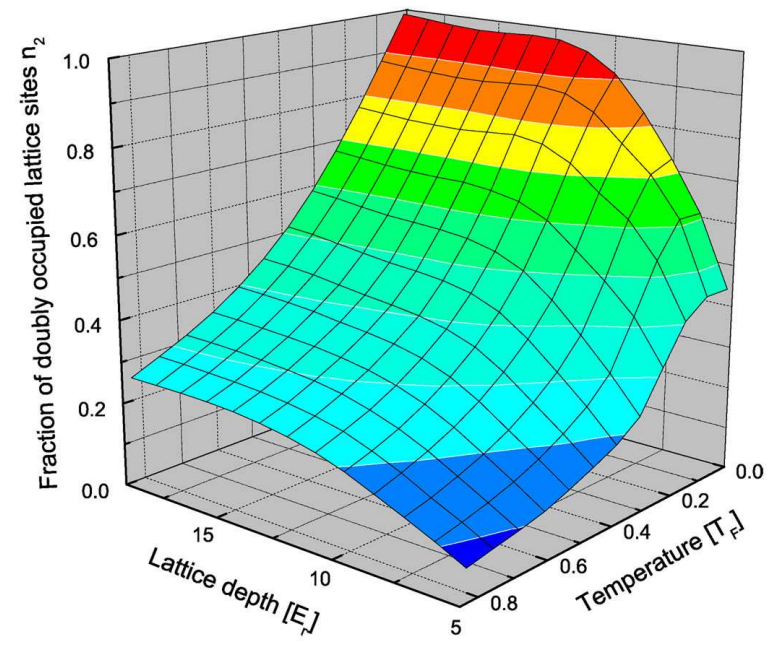

FIG. 3: Fraction of doubly occupied lattice sites $n_{2}$ in a threedimensional optical lattice as a function of the lattice depth and the temperature obtained by diagonalizing Hamiltonian (1). The particle number is $N=15000$ per spin state and the waist of the lattice laser is $w=70 \mu \mathrm{m}$, according to the situation in [7].

The dependence of the fraction of doubly occupied sites on temperature in three dimensions is stronger than in two dimensions but in both cases we find for $k_{B} T \ll E_{F}$ a predominantly linear dependence with a slope of order unity. For comparison we compute the fraction of doubly occupied lattice sites numerically. In figure $2 \mathrm{a}$ we compare the results in two dimensions with the exact analytical prediction of equation (7), which shows excellent agreement. In figure 2b the numerical results for a three-dimensional optical lattice are compared with the expression (12), which is valid for $k_{B} T \ll E_{F}$. We also find very good agreement in this situation. These results are also in agreement with the numerical results of [8]. 
When the system is not in the low-tunnelling regime we leave the range of validity of the analytic calculation. To quantify this limit, we have numerically studied how the fraction of doubly occupied lattice sites varies with the potential depth of the lattice (Fig. 3. We have chosen the experimentally realized values of $N=15000$ per spin state and a waist of $w=70 \mu \mathrm{m}$ [7] as parameters. From the calculations we observe that the analytical solution, which is independent of the lattice depth, is good for a potential depth $V \gtrsim 12 E_{r}$. In this low-tunnelling regime, $n_{2}$ depends on temperature only. For smaller values of the lattice depth numerical calculations are necessary to relate a measured fraction of doubly occupied lattice sites to the temperature.

Let us now compare our results with a recent experiment [9]. A noninteracting, two-component Fermi gas with an initial temperature in the harmonic trap of $T / T_{F} \simeq 0.25$ is loaded into a three-dimensional optical lattice. In a deep optical lattice $\left(V=15 E_{r}\right)$ molecules are created by adiabatically sweeping a magnetic field across a Feshbach resonance and the molecule fraction is determined to be $n_{2}=0.43$. From our theory we calculate a temperature of $T / T_{F}=0.46$ in the lattice. Provided that the sample in the lattice is in thermal equilibrium (which, however, has not been verified experimentally), this indicates a temperature increase of approximately a factor two as compared to the initial situation. This is expected for adiabatic loading of the optical lattice due to entropy conservation in the loading process.

Finally, we would like to address the weakly interacting two-component Fermi gas in an optical lattice. We take the on-site interaction between different spin states of strength $U$ into account using a mean-field approach [18], which requires that $k_{F}|a| \ll 1$. The occupation number $\nu_{\sigma}\left(r_{i}\right)$ per spin state $\sigma$ at a lattice site $r_{i}$ in the low-tunnelling regime is given by $\nu_{\sigma}\left(r_{i}\right)=\left(\exp \left[\left(V\left(r_{i}\right)+U \nu_{\uparrow}\left(r_{i}\right) \nu_{\downarrow}\left(r_{i}\right) / 2-\mu\right) / k_{B} T\right]+1\right)^{-1}$. This equation is solved self-consistently. The results show that the fraction of doubly occupied sites changes with the strength of $U$ : For repulsive interaction $n_{2}=\sum_{i} \nu_{\uparrow}\left(r_{i}\right) \nu_{\downarrow}\left(r_{i}\right) / N$ is diminished and for attractive interaction $n_{2}$ is increased. However, the interaction induced effect is smaller than the temperature effect. For $k_{F}|a|=0.05, w=70 \mu \mathrm{m}, N=$ 15000 (the parameters of figure 3) and a lattice depth of $25 E_{r}$ we observe a change of double occupancy by approximately $2 \%$, which is small as compared to the effect of temperature.

In conclusion, we have investigated a Fermi gas in an optical lattice with an underlying harmonic trapping potential. Our results have immediate consequences for ongoing experiments with ultracold fermions in optical lattices. We find that the Fermi gas is adiabatically heated by approximately a factor two when the gas is loaded into the lattice. Moreover, we calculate how the occupation of an optical lattice depends on temperature and find that the fraction of doubly occupied lattice sites for a noninteracting, two-component Fermi gas is a sensitive quantity for thermometry in the lattice.

We would like to thank T. Bourdel, C. Chin, T. Esslinger, K. Günter, H. G. Katzgraber, C. Kollath, H. Moritz, and T. Stöferle for helpful discussions.
[1] W. Hofstetter, J. I. Cirac, P. Zoller, E. Demler, M. D. Lukin, Phys. Rev. Lett. 89, 220407 (2002).

[2] F. Illuminati and A. Albus, Phys. Rev. Lett. 93, 090406 (2004).

[3] P. B. Blakie, A. Bezett, Phys. Rev. A 71, 033616 (2005).

[4] F. Werner, O. Parcollet, A. Georges, S. R. Hassan, Phys. Rev. Lett. 95, 056401 (2005).

[5] B. DeMarco, and D. S. Jin, Science 285, 1703 (1999).

[6] M. Rigol, A. Muramatsu, Phys. Rev. A 69, 053612 (2004).

[7] M. Köhl, H. Moritz, T. Stöferle, K. Günter, T. Esslinger, Phys. Rev. Lett. 94, 080403 (2005).

[8] H. G. Katzgraber, A. Esposito, M. Troyer, cond-mat/0510194 (2005).

[9] T. Stöferle, H. Moritz, K. Günter, M. Köhl, T. Esslinger, Phys. Rev. Lett. 96030401 (2006).

[10] T. Rom et al. Phys. Rev. Lett. 93, 073002 (2004).

[11] K. Xu et al., Phys. Rev. A 72, 043604 (2005).
[12] C. Ryu et al., cond-mat/0508201 (2005).

[13] C. Hooley, J. Quintanilla, Phys. Rev. Lett. 93, 080404 (2004).

[14] P. W. H. Pinkse et al., Phys. Rev. Lett. 78, 990 (1997).

[15] D. M. Stamper-Kurn et al., Phys. Rev. Lett. 81, 2194 (1998).

[16] K. Huang, Statistical Mechnanics, Wiley, 1987.

[17] G. Su, J. Chen, and L. Chen, Physics Letters A 315, 109 (2003).

[18] L. D. Carr, G. V. Shlyapnikov, Y. Castin, Phys. Rev. Lett. 92, 150404 (2004); L. D. Carr, R. Chiaramonte, M. J. Holland, Phys. Rev. A 70, 043609 (2004).

[19] M. Budde, K. Mølmer, Phys. Rev. A 70, 053618 (2004).

[20] G. Pupillo, C. J. Williams, N. V. Prokof'ev, Phys. Rev. A 73, 013408 (2006) .

[21] N. W. Ashcroft and N. D. Mermin, Solid States Physics, Saunders College Publishing, 1976. 